\title{
Criminologie
}

\section{Évaluer ce que fait la police : l'exemple australien}

\section{Benoît Dupont}

Volume 36, numéro 1, printemps 2003

Police et prévention : évaluation et analyse d'impact

URI : https://id.erudit.org/iderudit/006555ar

DOI : https://doi.org/10.7202/006555ar

Aller au sommaire du numéro

Éditeur(s)

Les Presses de l'Université de Montréal

ISSN

0316-0041 (imprimé)

1492-1367 (numérique)

Découvrir la revue

\section{Citer cet article}

Dupont, B. (2003). Évaluer ce que fait la police : l'exemple australien. Criminologie, 36(1), 103-120. https://doi.org/10.7202/006555ar

\section{Résumé de l'article}

Les réformes administratives ayant touché les services publics aux cours des vingt dernières années mettent résolument l'accent sur des modes de gestion entrepreneuriaux, qui privilégient les résultats et accordent une plus grande liberté quant aux moyens mis en oeuvre pour les atteindre. Les services de police n’ont pas échappé à cette tendance et, malgré les difficultés structurelles liées à l'évaluation des performances policières, de nouveaux instruments de mesure de l'efficacité et de l'efficience ont vu le jour. Ces derniers ont pour objectif de mettre à la disposition de la communauté des informations sur la qualité du service offert, mais également de favoriser l'identification et la diffusion des meilleures pratiques parmi les organisations policières. En s'appuyant sur l'exemple concret de l'outil développé en Australie depuis une dizaine d'années, on montrera quelle forme peuvent prendre ces nouveaux instruments, quelles sont leurs limites et comment ils pourraient être améliorés.
Ce document est protégé par la loi sur le droit d'auteur. L'utilisation des services d’Érudit (y compris la reproduction) est assujettie à sa politique d'utilisation que vous pouvez consulter en ligne.

https://apropos.erudit.org/fr/usagers/politique-dutilisation/ 


\title{
Évaluer ce que fait la police: l'exemple australien
}

\author{
Benoît Dupont \\ Research Fellow \\ National Centre for Security and J ustice \\ Australian National University - Research School of Social Sciences
}

Depuis juin 2002

Professeur

École de criminologie

Université de Montréal

benoit.dupont@umontreal.ca

RÉSUMÉ - Les réformes administratives ayant touché les services publics aux cours des vingt dernières années mettent résolument l'accent sur des modes de gestion entrepreneuriaux, qui privilégient les résultats et accordent une plus grande liberté quant aux moyens mis en œuvre pour les atteindre. Les services de police n'ont pas échappé à cette tendance et, malgré les difficultés structurelles liées à l'évaluation des performances policières, de nouveaux instruments de mesure de l'efficacité et de l'efficience ont vu le jour. Ces derniers ont pour objectif de mettre à la disposition de la communauté des informations sur la qualité du service offert, mais également de favoriser l'identification et la diffusion des meilleures pratiques parmi les organisations policières. En s'appuyant sur l'exemple concret de l'outil développé en Australie depuis une dizaine d'années, on montrera quelle forme peuvent prendre ces nouveaux instruments, quelles sont leurs limites et comment ils pourraient être améliorés.

ABSTRACT - Over the past twenty years, government services have been swept by a wave of public reforms, which emphasise entrepreneurial management techniques, place a high priority on the delivery of outcomes and devolve more power to managers in order to achieve this end. Police services have not escaped this trend, and despite the structural difficulties linked to police performance evaluation, new effectiveness and efficiency measurement tools have been designed. Their objective is to provide information on the quality of service delivery to the community, but also to facilitate the identification and diffusion of best practices among police organizations. Relying on the example of the methodology developed in Australia over the past ten years, we will show the design of these new instruments, what their limitations are and how they could be improved. 
Parmi les nombreux dilemmes associés à l'institution policière, celui de l'évaluation figure au premier plan. L'impact de l'activité policière est structurellement difficile à mesurer et la plupart des instruments de mesure développés à cet effet souffrent, par conséquent, de nombreuses limitations. D 'une part, les méthodes qui cherchent à évaluer l'efficacité de la police, tant sur le plan de la réduction de la délinquance que de la diminution du sentiment d'insécurité, sont confrontées à une multiplicité de facteurs externes susceptibles d'influencer les données dans une mesure difficile à prédire. Ainsi, une baisse de la délinquance peut aussi bien être attribuée à une meilleure organisation du travail policier qu'à une embellie économique ou à une réforme législative qui décriminalise certains comportements illégaux. II est impossible, dans ce contexte, d'identifier des indicateurs d'efficacité qui permettent d'évaluer l'impact de l'activité policière isolément de ces autres facteurs. D'autre part, les méthodes qui tendent à évaluer l'efficience de la police se concentrent sur des procédures et des processus qui n'ont qu'un rapport partiel avec l'impact souhaité: les taux d'élucidation, par exemple, ne peuvent être pris en considération que dans la mesure où les taux de déclaration et d'enregistrement sont connus (Wilson, 1993). Les difficultés créées par ce dilemme sont accentuées par une dichotomie fonctionnelle inhérente au travail de la police, qui opère une distinction entre sa dimension répressive et préventive. Les activités de prévention, dont l'impact semble plus durable que celui des mesures de répression, se caractérisent par une absence d'événements quantifiables. Ce qui ne se produit pas est par définition impossible à mesurer, à moins d'arriver à concevoir des outils de simulation qui soient suffisamment fiables. De plus, l'émergence de nouvelles agences gouvernementales et de réseaux d'acteurs prenant une part active aux initiatives de prévention de la délinquance introduisent un élément supplémentaire de complexité qui est encore trop rarement pris en compte.

M algré ces difficultés, l'évaluation du travail policier fait l'objet, depuis une vingtaine d'années, d'un intérêt croissant, qui se traduit notamment par l'élaboration de nouveaux outils de mesure. Cette ardeur évaluatrice a pour cadre le mouvement de réforme de l'État et des services publics qui a touché les démocraties libérales, rendant obsolète le modèle de l'État providence au profit d'un État régulateur minimaliste. 0 utre des politiques économiques rejetant toute notion d'interventionnisme étatique, ce virage néolibéral pris par les gouvernements des sociétés postindustrielles se traduit par une nouvelle approche de la gestion 
publique. Le modèle d'administration monolithique et pléthorique a cédé la place à une administration entrepreneuriale appliquant les principes de gestion des grandes entreprises multinationales. 0 n peut identifier dans de nombreux pays une remarquable similarité quant aux traits saillants de cette nouvelle approche. 0 n note, d'abord, une nouvelle liberté accordée aux gestionnaires de l'action publique afin de remplir au mieux la mission de satisfaction des citoyens, transformés en consommateurs. L'importance accordée aux résultats, ou en d'autres termes le primat des fins sur les moyens, a ensuite requis la mise en place de critères explicites de mesure des performances en termes de rentabilité (rendement, ou «ouputs»), d'efficacité (gestion par analyse de résultat, ou « outcomes»), mais aussi de satisfaction du public (Cheung, 1997 : 516-525).

Les services de police n'ont pas été épargnés par cette déferlante néolibérale et ont dû justifier leurs dépenses auprès de gouvernements en quête de rationalisations budgétaires. Le «baromètre» traditionnel que représentaient jusque-là les statistiques officielles de la délinquance, leur évolution annuelle et les taux d'élucidation par catégorie d'actes délictueux ont cessé d'être considérés comme un instrument suffisamment fiable de mesure de l'efficacité du travail policier. 0 utre la propension des services de police à présenter ces statistiques sous un jour flatteur (Loveday, 1999 : 356 ; 2000 : 224), voire à les manipuler, ce qui est d'autant plus aisé qu'ils possèdent souvent un monopole sur leur production, ces instruments de mesure sont particulièrement exposés aux dilemmes présentés plus haut. Tout d'abord, les taux d'élucidation dépendent indirectement de la nature des délits déclarés à la police par leurs victimes et I'on sait que certains d'entre eux ont une probabilité plus élevée que les autres d'aboutir à l'identification de leurs auteurs. Le pourcentage du taux d'élucidation pouvant être exclusivement attribué aux capacités de détection et d'investigation de la police est ainsi évalué aux environs de $3 \%$, les $97 \%$ qui restent reposant sur les renseignements fournis par les victimes ou les témoins (Loveday, 1993 : 142). Ensuite, une portion relativement négligeable du temps policier est consacrée à la lutte contre la criminalité (Bennett et Lupton, 1992; Bayley, 1994 ; PA Consulting Group, 2001) et I'utilisation des taux d'élucidation comme outil exclusif d'analyse de l'efficacité conduit à ignorer tout le pan préventif des activités policières. Ce constat est d'autant plus problématique qu'on a vu se développer ces dernières années une division et une spécial isation croissantes du travail policier avec, d'un côté, des stratégies répressives d'intervention regroupées sous l'appellation de Police de tolérance zéro et, 
de l'autre côté, une volonté de tisser des liens durables avec les communautés qui constituent l'environnement policier, volonté qui se traduit par le développement d'initiatives de police de proximité.

De nouveaux outils d'évaluation ont alors été conçus afin de mieux prendre en compte les diverses facettes du travail policier, mais aussi de renforcer le contrôle budgétaire des services de police. À travers l'exemple de l'Australie, qui expérimente depuis une dizaine d'années un tel système d'évaluation des performances de ses services publics, on montrera quelle forme peuvent prendre ces instruments de mesure de deuxième génération. Leurs limites méthodologiques et les effets contre-intuitifs qu'ils peuvent entraîner seront analysés, avant que des propositions visant à leur perfectionnement soient esquissées.

\section{Le contexte politique de l'évaluation des services publics australiens}

En Australie, le constat du déficit méthodologique des méthodes traditionnelles d'évaluation des performances policières est à l'origine de l'élaboration d'un outil national modernisé. Cette démarche n'est d'ailleurs pas limitée aux organisations policières, puisqu'elle s'étend à de nombreux autres services publics. L'approche australienne de l'évaluation des performances policières repose moins sur une prescription de résultats à atteindre, comme c'est le cas en Angleterre avec les «B est value performance indicators» instaurés par le Local G overnment A ct de 1999, que sur une mise en confrontation des différents services de police par le biais de leurs performances, indicateur par indicateur. La structure fédérale de l'État australien est à l'origine de cette approche, moins directive mais tout aussi rigoureuse. La Constitution australienne confère en effet aux États fédérés une compétence exclusive sur l'administration de la police et de la justice. Par conséquent, chaque État dispose d'une force de police centralisée, d'une administration judiciaire et d'un service pénitentiaire qui opèrent indépendamment de leurs homologues des autres États, dans un cadre législatif qui leur est propre. Au niveau national, une force de police fédérale dispose d'une compétence limitée dans les domaines de la lutte contre la délinquance en col blanc et le crime organisé.

L'emprise réduite du pouvoir fédéral dans les affaires de police et de justice, le besoin manifeste d'un cadre d'évaluation plus rigoureux que les traditionnelles statistiques de la délinquance et un souci d'homogénéisation des indicateurs de performance ont conduit à la mise en place de 
mécanismes de collaboration entre les gouvernements de chaque État. En 1993, le premier ministre australien et ses homologues des six États et des deux Territoires décidèrent de créer un mécanisme national d'évaluation de la qualité des services fournis au public par les administrations fédérales et locales ${ }^{1}$. Dans le cas des politiques publiques de sécurité et des services de police, la comparaison entre des services identiques offerts dans différentes juridictions a pour objectif principal la fin du monopole de l'information que détiennent les administrations locales. Chaque communauté ne possédant qu'un service de police, il lui est difficile d'obtenir une information qui ne soit pas produite, et incidemment contrôlée, par cette agence unique.

Dans la logique du marché qui a alimenté les réformes managériales des services publics, la comparaison des résultats et de l'efficacité des institutions monopolistiques a alors été conçue comme une alternative aux incitations qui existent sur les marchés compétitifs. Les «clients», qu'il s'agisse des décideurs politiques ou des électeurs, disposent ainsi en théorie d'informations leur permettant de faire pression sur les services publics et de renforcer leur degré d' «accountability », les résultats inférieurs à la moyenne nationale étant dorénavant disponibles de manière standardisée, et publiés annuellement. N éanmoins, si les statistiques des performances sont mises à la disposition de chaque citoyen qui le désire, ceux-ci ne disposent pas des même options que celles qui sont à la disposition des consommateurs ou des actionnaires. La migration vers une autre juridiction est souvent onéreuse, voire impossible, et les échéances électorales représentent un mode de contrôle trop peu fréquent et imparfait (Smith, 1992). Cet outil est donc en priorité un instrument de contrôle par les ministres de l'efficacité et de l'efficience des services de police qu'ils dirigent «à distance». Il leur procure une «expertise» et une interprétation indépendantes de celles que leur présentent les chefs de la police.

Avant de poursuivre, il est nécessaire de s'attarder à quelques éléments de terminologie. D errière le paravent de l'éval uation sont regroupés une

1. Bien que chaque domaine d'activité relève potentiellement de cette initiative, il fut décidé, dans un premier temps, que seuls huit champs de compétence seraient pris en considération : les services hospitaliers, l'enseignement primaire et secondaire, la formation professionnelle, le logement social, les services aux personnes et aux familles en difficulté, l'administration judiciaire et les services de police et correctionnels. Ces services, qui représentent plus du tiers des dépenses publiques du Commonwealth et des États, ont été choisis en raison de I'homogénéité de leurs objectifs d'une juridiction à l'autre, du manque de données statistiques permettant une mesure comparative de leurs performances et de leur contribution à l'économie nationale et au bien-être de la communauté. 
myriade de termes qui doivent être définis avec soin. Le concept fondamental est ici celui d'efficacité, qui quantifie l'adéquation entre les effets attendus et effectifs d'un programme et les objectifs ou buts opérationnels d'une politique (Crawford, 1998: 53). L'effectivité ou efficience, par contraste, ne fait référence qu'au processus de production des biens ou des services et aux ressources utilisées à cette fin, sans que la nature des résultats atteints soit un facteur déterminant. Le premier concept aurait ainsi pour axe central les fins, alors que le second ne se préoccuperait que des moyens. Comme le souligne Grabosky (1988 : 2), un service de police idéal devrait être à la fois efficace et efficient. La réalité est souvent tout autre et un service de police pourra s'avérer efficace au prix de dépenses pharaoniques, alors qu'un autre service pourra faire preuve de frugalité sans produire de résultat visible. Ainsi, la mobilisation d'unités policières spécialisées dans le maintien de l'ordre peut s'avérer dans certaines circonstances un outil de dissuasion efficace, muselant les tentations de recours à la violence lors des manifestations de rue. Toutefois, une approche négociée avec les organisateurs de ces manifestations peut produire un résultat identique tout en faisant l'économie des ressources policières. A contrario, l'émission d'un plus grand nombre d'amendes de stationnement par un service de police traduit certainement une baisse du coût de production unitaire de chacune, mais n'améliore en rien la sécurité routière des citoyens ou ne réduit pas le coût pour la communauté des accidents de la route. La productivité- ou l'économie - est alors la mesure de l'efficacité de l'organisation pour une quantité de ressources données, faisant le lien entre les concepts d'efficacité et d'efficience. La distinction entre les deux termes est purement d'ordre sémantique (M oore, 1992: 43). Cette mesure peut prendre la forme d'une analyse respective des coûts et des bénéfices d'une activité afin de déterminer si les ressources utilisées produisent une valeur équivalente ou supérieure, ou au contraire sont gaspillées et pourraient être plus utiles ailleurs. L'analyse coûts-efficacité cherche pour sa part à déterminer de quelle manière un objectif spécifique peut être atteint au moindre coût, l'opportunité de ce dernier ne faisant pas débat. Si cette démarche paraît relativement facile à concrétiser dans le cas de services publics remplissant des fonctions simples à formaliser, des difficultés émergent dans le contexte plus complexe des activités de police. 


\section{Le rapport annuel sur les services publics: un outil d'évaluation comparatif, évolutif et intégrateur}

Le modèle d'évaluation australien est piloté depuis 1993 par un groupe de travail comprenant les représentants des différents services de police et présidé par le directeur de l'administration du Trésor de la N ouvelleGalles du Sud. Après de longues discussions, ce groupe de travail a développé une liste d'indicateurs d'efficacité et d'efficience considérés comme représentatifs de l'activité policière. La particularité de ce modèle, outre sa dimension comparative, est qu'il est considéré par ses créateurs comme évolutif et intégrateur. Le caractère évolutif découle du souhait de voir les indicateurs refléter les prio rités stratégiques et les besoins changeants de la société et des services de police, particulièrement dans le domaine de l'efficience, mais également de prendre en compte les innovations des sciences sociales quant à la mesure des phénomènes complexes. L'intégration de données provenant non seulement des services de police, mais également de sources externes telles que les administrations du Trésor, le Bureau national de la statistique et certains organes de sondages privés permet de surcroît à ce modèle de revendiquer une diversité des sources et une rigueur méthodologique qui font défaut aux instruments de mesure traditionnels.

La dernière mouture du modèle d'évaluation comporte 29 indicateurs de performances prenant en compte à la fois l'efficacité (22 indicateurs) et l'efficience (7 indicateurs) du travail policier (Steering Committee for the Review of Commonwealth/State Service Provision, 2002)2. Ces derniers sont répartis en fonction des quatre groupes d'activités policières identifiés par le comité de pilotage: sécurité publique générale, investigations criminelles, sécurité routière et soutien à la procédure judiciaire. Pour les activités de sécurité publique, les indicateurs d'efficacité comprennent par exemple le nombre de délits déclarés, les taux de déclaration à la police, les taux de victimisation, le sentiment d'insécurité et la préoccupation à l'égard de la délinquance. L'indicateur d'efficience indique le coût de l'offre du service de sécurité publique par habitant de l'État considéré afin de prendre en considération les variations démographiques. Une démarche similaire est adoptée pour les trois autres catégories d'activités.

2. Les chapitres relatifs au fonctionnement des tribunaux et des services correctionnels sont pareillement détaillés. 
TABLEAU 1

Indicateurs nationaux de performance employés en 2001

\begin{tabular}{|c|c|c|}
\hline \multirow[t]{2}{*}{$\begin{array}{l}\text { Sécurité } \\
\text { générale }\end{array}$} & Efficacité & $\begin{array}{l}\text { - Sentiment d'insécurité (au domicile, dans les endroits } \\
\text { publics et les transports en commun) } \\
\text { - Préoccupation à l'égard de la délinquance (par type } \\
\text { d'infraction) } \\
\text { - Crimes déclarés } \\
\text { - Taux de déclaration } \\
\text { - Taux de victimisation }\end{array}$ \\
\hline & Efficience & - Coût du service offert en dollars par habitant \\
\hline \multirow[t]{2}{*}{ Investigations } & Efficacité & $\begin{array}{l}\text { - Résultat des enquêtes : crimes contre les personnes et } \\
\text { crimes contre les biens (taux d'enquêtes finalisées } \\
\text { dans les } 30 \text { jours après enregistrement par type } \\
\text { d'infraction) } \\
\text { - Taux de recouvrement des véhicules volés }\end{array}$ \\
\hline & Efficience & - Coût du service offert en dollars par habitant \\
\hline \multirow[t]{2}{*}{$\begin{array}{l}\text { Sécurité } \\
\text { routière }\end{array}$} & Efficacité & $\begin{array}{l}\text { - Taux autodéclaré d'utilisation de la ceinture de } \\
\text { - Técurité } \\
\text { - Taux autodéclaré de conduite sous l'emprise de l'alcool } \\
\text { - Morts sur la route } \\
\text { - Hospitalisations causées par des accidents de la route } \\
\text { - Préoccupation à l'égard de la sécurité routière }\end{array}$ \\
\hline & Efficience & $\begin{array}{l}\text { - Coût du service offert en dollars par habitant } \\
\text { - Coût du service offert en dollars par voiture } \\
\text { immatriculée }\end{array}$ \\
\hline \multirow[t]{2}{*}{$\begin{array}{l}\text { Procédure } \\
\text { pénale }\end{array}$} & Efficacité & $\begin{array}{l}\text { - Décès en détention provisoire (populations générale } \\
\text { et autochtone) } \\
\text { - Proportion d'affaires où les accusés sont jugés } \\
\text { coupables (crimes et délits) } \\
\text { - Proportion de mesures de médiation offertes à des } \\
\text { délinquants mineurs }\end{array}$ \\
\hline & Efficience & $\begin{array}{l}\text { - Coût du service offert en dollars par personne } \\
\text { (procédure pénale et détention provisoire) } \\
\text { - Coût des dommages et intérêts versés par la police } \\
\text { dans le cadre de poursuites abusives }\end{array}$ \\
\hline
\end{tabular}

Bien entendu, certains indicateurs ne sont pas réductibles à l'un de ces quatre groupes et rendent plutôt compte d'une performance globale de l'organisation et de ses membres. On peut ranger dans cette catégorie le niveau de satisfaction des usagers, l'intégrité et le professionnalisme avec lesquels les agents s'acquittent de leurs tâches, ou encore le degré d'équité dans l'accès aux emplois offerts aux femmes et aux minorités. 
L'un des intérêts de ce modèle est la volonté de lier les intrants aux extrants dans un domaine de l'activité administrative réputé pour sa complexité. $\mathrm{N}$ ous avons vu que les indicateurs tentent de prendre en considération aussi bien le coût des services offerts que l'efficacité réelle et symbolique de la police. Par efficacité réelle, on entend l'impact sur la réduction de la délinquance et des accidents de la route, ou encore sur l'augmentation des taux de condamnation des suspects présentés devant les tribunaux. Par efficacité symbolique, il est fait référence à la perception subjective du sentiment d'insécurité ou à la confiance accordée à la police en tant qu'institution de contrôle social. Si les budgets alloués chaque année par les gouvernements aux services de police et le financement par ces derniers de programmes spécifiques correspondant aux quatre grandes catégories d'activités sont relativement faciles à connaître, il n'en est pas de même en ce qui concerne l'activité quotidienne des agents. U ne mesure précise de cette dernière est pourtant essentielle afin de mieux comprendre les mécanismes par lesquels les intrants sont transformés en extrants, ou ce qui caractérise l'efficacité de certaines stratégies innovantes.

En effet, dans le cas du travail policier, la différenciation fonctionnelle qui touche les unités spécialisées d'enquête, de police scientifique ou de police de la route ne s'applique pas aux agents en uniforme qui accomplissent une multiplicité de tâches, partiellement définies par leur hiérarchie, mais également largement déterminées par les appels du public. Ce travail peut être appréhendé comme une boîte noire dont il est indispensable de connaître le mécanisme interne si l'on veut comprendre comment elle atteint ses objectifs ou au contraire échoue dans cette tâche (Pawson et Tilley, 1997). Si I'on peut quantifier de manière générale, à l'aide des intrants et des extrants, les succès de la police, la détermination de ce qui dans les pratiques quotidiennes fait ce succès et du coût qui y est associé ne peut s'effectuer que par le biais d'un recueil de données sur les activités des agents, qu'il s'agisse de répression proactive, réactive ou de prévention.

Plusieurs méthodes sont utilisées par les services australiens. Tout d'abord, le sondage d'activité permet d'enregistrer sur une courte période - en général deux ou trois semaines - les tâches et les activités menées par les agents durant leur journée de travail. L'estimation du temps passé à chaque tâche est alors comparée avec les intrants utilisés afin de déterminer les coûts afférents à chaque activité. Une deuxième méthode, celle des feuilles d'activité ou «time sheets», requiert des agents 
qu'ils indiquent pour chaque période d'une demi-heure l'activité menée, à l'aide de codes prédéterminés. Cette méthode est plus précise, dans la mesure où elle donne une image des activités de l'organisation à un moment donné. Enfin, la troisième méthode est celle des emplois du temps distribués aux agents au début de leur période de travail et qui sont remplis en fonction des tâches effectuées afin de tenir compte à la fois des activités réactives d'assistance transmises par radio et des activités proactives entreprises à l'initiative des agents. Le développement de la mesure de l'activité policière est lié aux progrès des technologies de collecte, de «stockage» et de traitement de l'information, et plus particulièrement de l'informatique. Dans le contexte actuel, les données recueillies servent principalement à évaluer les coûts de chaque type d'activités policières, mais on peut imaginer sans difficulté leur mise à contribution pour des évaluations plus ciblées axées sur l'efficacité des différentes stratégies d'intervention de la police.

\section{Quelques limites méthodologiques du modèle australien}

M algré la complexité du modèle mis en œuvre, un examen critique des méthodes de collecte de l'information, de la qualité des données assemblées et de l'interprétation des résultats obtenus met en lumière les obstacles qui restent à surmonter pour faire de cet outil un instrument d'évaluation des performances policières qui ne se limite pas au cadre d'un simple audit financier.

En ce qui concerne la nature et la qualité des informations nécessaires à cet exercice d'évaluation des performances, certaines limites peuvent être identifiées. D ans le modèle australien, les résultats sont statistiquement définis isolément les uns des autres. C'est par exemple le cas des taux d'élucidation par types de délits, du sentiment d'insécurité du public ou de la confiance accordée par la population à sa police. Pourtant, la logique opérationnelle du travail policier est à l'origine de relations bien plus complexes entre les effets produits, plusieurs conséquences distinctes pouvant être enregistrées à l'issue d'une intervention unique. C'est par exemple le cas lorsqu'une arrestation sur la voie publique contribue accessoirement à rassurer les passants qui en sont les témoins sur l'efficacité de la police et à empêcher que le suspect interpellé ne commette d'autres infractions pendant la durée de sa détention. II est structurellement difficile d'établir un lien direct entre les ressources utilisées et des résultats défi- 
nis, dont on ne connaît par ailleurs pas toute l'étendue. Il en est de même de la qualité des résultats obtenus: les activités proactives de patrouille diffèrent par leur impact sur le sentiment d'insécurité selon qu'elles sont pédestres ou motorisées. Le coût horaire est pourtant le même en terme de salaires et la productivité en terme du nombre d'arrestations effectuées dans les deux modes de patrouille pourra être identique.

La difficulté à quantifier autrement que grossièrement la facette proactive du travail policier, qui, de l'aveu même des gestionnaires, représente approximativement $40 \%$ de celui-ci en Australie (Steering Committee for the Review of Commonwealth/ State Service Provision, 1997 : 104), soustrait à toute évaluation des performances une fraction substantielle de l'activité considérée. $D$ e même, en ce qui concerne le versant réactif du travail policier, cette méthode d'évaluation est limitée aux incidents enregistrés pour lesquels des informations statistiques sont disponibles. Plus qu'à la victimisation non déclarée, qui peut être mesurée avec un certain degré de précision par le biais de sondages, on fait ici allusion aux incidents portés à la connaissance de la police, mais ne donnant lieu à aucun enregistrement. L'une des contributions capitales des études sociologiques sur la police fut d'avoir démontré, dès les années 1960, le pouvoir discrétionnaire dont disposent les policiers dans leur travail quotidien et leur mise en œuvre de modes de résolution informels des délits dont ils sont témoins (Waddington, 1999 : 31). Certains incidents sont par exemple résolus par le biais d'une simple mise en garde verbale des coupables, s'il s'avère que le délit commis est anodin et que l'avertissement est jugé suffisant par les policiers. Des querelles de voisinage peuvent également être désamorcées par les négociations d'agents appelés sur les lieux, évitant là encore qu'une procédure soit engagée. Plus prosaïquement, à l'approche du changement d'équipe, des policiers choisiront d'ignorer un délit commis sous leurs yeux afin de ne pas se retarder, ou au contraire, se mettront activement «en chasse» de la moindre infraction susceptible de leur procurer quelques heures de travail supplémentaires par le traitement bureaucratique qu'elle nécessitera. La mesure effective des réponses policières à l'ensemble des incidents traités est de la sorte faussée par l'exercice de ce pouvoir discrétionnaire qui confère aux agents la latitude de ne pas enregistrer tous les incidents constatés et, quand ils le font, celle de ne pas avoir à justifier la réponse qu'ils y ont apportée. Ce problème est renforcé par l'informatisation du travail policier et par la tendance à la simplification des formulaires d'enregistrement des plaintes et des incidents constatés, qui délaissent la narration libre au 
profit de questions à choix multiples censées assurer une homogénéisation des informations recueillies (Ericson et Haggerty, 1997 : 382). Si les données recueillies sont sans aucun doute plus complètes, elles manquent néanmoins de profondeur en comparaison des rapports plus circonstanciés du passé. Les instruments de mesure de l'activité apportent une solution partielle à ce problème, mais ils n'éliminent pas totalement les conséquences du facteur discrétionnaire, qui peut mener les agents à une occultation volontaire ou à un « oubli » de certaines activités lors de la collecte des données.

L'étendue des informations recueillies n'est d'ailleurs pas la seule source de faiblesse de cette méthode d'évaluation. La qualité de certaines informations doit encore être améliorée. Tout d'abord, le travail des employés administratifs non assermentés ne fait pas l'objet de renseignements détaillés, bien qu'ils représentent $20 \%$ des effectifs des services de police australiens et qu'ils contribuent à ce titre pour une part non négligeable aux résultats de ces derniers. Les personnels policiers en arrêts-maladie prolongés ne sont pas pris en compte, réduisant quelque peu l'efficience théorique du commissariat dans lequel ils sont plus nombreux que la moyenne. Cette dimension est par contre présente dans le modèle anglais d'évaluation des performances (Secretary of State, 2002). Les informations relatives aux arrestations sont également frappées d'un certain degré d'imprécision. Les statistiques des arrestations attribuées à chaque service sont « enrichies» des arrestations effectuées dans sa juridiction administrative par d'autres agences spécialisées dans la lutte contre le crime organisé, telles que la police fédérale ou la «C rime Commission » en N ouvelle-Galles du Sud. Les arrestations abusives ou erronées sont également prises en compte comme un résultat positif (Steering Committee for the Review of Commonwealth/ State Service Provision, 1997 : 105), malgré l'indication d'une inefficacité indéniable de l'organisation.

L'interprétation des résultats, pour sa part, entraîne des questions quant à la validité des liens de causalité inférés. Le problème soulevé en introduction, relatif à l'absence de résultats mesurables des activités proactives ou préventives rend une évaluation exhaustive du travail policier improbable et contient également le germe d'une erreur d'interprétation des résultats obtenus. Les performances d'un service dont les activités sont majoritairement liées à la détection du crime sont, dans ce modèle, jugées supérieures à celles d'un service allouant une part importante de ses ressources à des programmes de prévention dont les répercussions 
sont par définition invisibles puisque c'est à la prophylaxie des actes délictueux - et non à leur répression - qu'ils s'attachent.

Enfin, l'environnement opérationnel et social des organisations évaluées est porteur de différences qui rendent les comparaisons directes difficilement exploitables. 0 n peut répertorier six facteurs qui contribuent à des écarts significatifs de performances entre les organisations évaluées. M algré la similitude du travail policier d'une juridiction à l'autre, les services de police peuvent être investis de responsabilités différentes, particulièrement dans le domaine de la prévention de la délinquance, où les initiatives locales et les programmes de partenariat avec d'autres services publics s'articulent à travers une multitude de configurations. Plus précisément, la nouvelle gouvernance nodale qui caractérise les sociétés contemporaines entraîne des modifications structurelles, qui ont notamment pour conséquence de délester la police de certaines de ses responsabilités en tant que fournisseur exclusif de «sécurité» (Kempa et al., 1999 ; B ayley et Shearing, 2001). Cette transformation n'est pas sans produire des répercussions visibles sur les performances de l'organisation policière, qui doivent dorénavant être appréhendées comme le résultat d'un travail en réseau.

Les organisations policières peuvent égal ement poursuivre des objectifs variés et distincts d'un État à l'autres. L'arrivée à la tête de la police de N ouvelle-Galles du Sud d'un commissaire anglais a orienté le travail de cette force vers des stratégies de renseignement criminel et de «police d'expertise» coûteuses en investissements technologiques. Les services de police des États moins exposés à une délinquance organisée, comme la Tasmanie ou le Territoire du Nord, font pour leur part face à d'autres priorités ou à d'autres défis, tels que l'absence d'économies d'échelle suffisantes pour permettre l'adoption de technologies ou de stratégies novatrices. Les facteurs géographiques et démographiques jouent également un rôle important sur les besoins exprimés auprès de la police et sur les modes par lesquels celle-ci répond à ces besoins. La police d'Australie-0 ccidentale opère ainsi sur la juridiction la plus étendue du monde, avec une surface de plus de deux millions et demi de kilomètres carrés couverte par seulement 4700 policiers ${ }^{3}$, ce qui augmente de façon considérable les coûts liés à l'offre de services par la police. D'autres juridictions, comme le Territoire du Nord, ont la responsabilité d'une population majoritairement autochtone, qui requiert la mise en œuvre de programmes

3. Australian Bureau of Statistics, 1997. 
de coproduction de sécurité avec les communautés isolées du centre, dont les modes de régulation traditionnels doivent être pris en considération. De telles variations doivent être prises en compte par les gouvernants qui voudraient comparer l'efficience de leur force de police à l'aune de celle des États jugés «performants» ou innovateurs en ce domaine, au risque de conduire à des conclusions erronées (Smith, 1992: 16).

\section{Les effets contre-intuitifs}

Au-delà des limites techniques énumérées plus haut, et qui peuvent être levées au fil du temps par un perfectionnement de la méthode employée, un écueil important à toute tentative d'évaluation réside dans le mythe de neutralité qui l'accompagne. Loin de conduire passivement à une amélioration des performances par la publication des résultats de chacun des services concernés, le processus décrit ici contribue, à l'instar de tous les autres outils d'évaluation, à une altération des pratiques de ceux qui y sont soumis, dans une mesure qu'il est irréaliste de vouloir quantifier. Ce modèle pousse imperceptiblement la police à n'entreprendre que des activités mesurables, qui se traduisent par des données pouvant être aisément recueillies et stockées, et qui n'offrent, en fin de compte, qu'une image appauvrie ou dénaturée de la qualité du travail policier (Loveday, 1993: 143).

Ce fut le cas au cours des années 1970 et 1980, lorsque la réduction du temps de réponse aux appels d'urgence a semblé être la panacée en matière de détection des infractions et de leurs auteurs (qui n'auraient pas le temps de quitter les lieux du crime si un véhicule de police pouvait être envoyé sur place en quelques minutes) ${ }^{4}$. C'est de nouveau le cas avec des indicateurs de performance plus complexes. En Australie comme aux États-Unis, de nombreux scandales ont révélé les conséquences de l'obsession pour la mesure en temps réel des statistiques et son articulation avec la promotion et la rémunération des officiers supérieurs de police. La reclassification de crimes violents en délits, l'effacement de certains crimes des bases de données ou la délocalisation de crimes à l'extérieur

4. En 1998, I'arrivée des technologies de positionnement par satellite (GPS), d'allocation des appels informatisée et des systèmes d'information géographique (SIG ) ont remis au goût du jour cette religion de l'intervention rapide comme panacée à l'amélioration de l'efficacité policière (The Audit 0 ffice of N ew South Wales, $1998: 61$ ). 
de la zone de compétence ${ }^{5}$ sont autant de stratagèmes employés afin de contenir les statistiques dans le carcan fixé par les normes d'évaluation des performances.

Nick Tilley identifie de la même façon huit effets contre-intuitifs à I'utilisation abusive des indicateurs de performances: l'étroitesse de vue restreinte à ce qui est quantifiable; la «suboptimisation » qui amène à une moindre qualité de service par une concentration sur des activités définies de façon restrictive; la «myopie» qui empêche de prendre en considération les objectifs à plus long terme; la «fixation» sur la mesure plutôt que sur ce qui doit être mesuré; la corruption délibérée des données; I'acceptation de l'interprétation des résultats sans approche critique; les comportements de «jeu» qui comprennent la gestion stratégique des comportements tels que la sous-performance délibérée; "|'ossification», ou la poursuite inflexible des objectifs de performance définis à un moment donné; la «démoralisation» des travailleurs qui offrent un service dont la mesure est inadaptée ou inexistante - particulièrement pertinente dans le cas des activités de prévention de la criminalité- et le scepticisme public renforcé par le sabotage des employés (Tilley, 1995 : 4-5).

La politisation du travail de la police et de ses résultats génère de surcroît un effet de retour qui amoindrit la fiabilité des indicateurs de performance. Par un effet de rétroaction négative («negative feedback»), on peut fréquemment observer une corrélation positive entre le niveau des ressources (intrants) et la faiblesse des performances. En effet, des évaluations catastrophiques sont susceptibles d'attirer l'attention de l'opinion publique et des décideurs politiques sur les problèmes local isés de délinquance ou d'intégrité policière. En règle générale, la réponse politique consiste en une allocation d'urgence de ressources financières et humaines afin de pallier ces problèmes, alors que ces dernières auraient pu être utilisées ailleurs à meilleur escient, ce qui produit des distorsions importantes dans une perspective d'analyse temporelle. La dimension politique, dont l'existence n'est pas prise en compte par l'outil d'évaluation malgré la contrainte indéniable qu'elle exerce sur les performances policières, nous amène à soulever la question plus générale de l'usage qui est fait des résultats obtenus.

5. Ce fut le cas du chef de la police des transports en commun de la ville de N ew York, qui a dû démissionner en 1998 après qu'il fut prouvé qu'il avait mis en place un système élaboré afin de "transférer » les crimes commis dans le métro en crimes commis dans la rue, dont la responsabilité était ainsi transmise à un autre service que le sien. 
Les critiques formulées plus haut quant au manque de prise en considération des particularités politiques et organisationnelles de l'institution policière dans l'évaluation de ses performances ne doivent pas occulter les mérites de cet outil novateur. La richesse des données recueillies à travers une stratégie de sources multiples et une constante résolution d'améliorer les méthodes d'analyse traduisent une volonté indéniable d'offrir aux électeurs et aux décideurs politiques des indicateurs aussi complets que possible. N éanmoins, la conception et la structuration des indicateurs sous l'égide de la «Productivity Commission» indique une préoccupation dominante pour l'efficience et laisse entrevoir l'emprise des administrations du Trésor sur cet outil. La comparaison systématique des coûts de chaque type d'activité policière offre à ces dernières les moyens de pression nécessaires sur les organisations policières afin que celles-ci réduisent ou contrôlent leurs dépenses. Une telle approche est certainement justifiée par l'intérêt des contribuables, mais réduit à l'extrême la complexité structurelle et fonctionnelle de l'offre publique de sécurité. La publication des résultats annuels donne bien lieu à l'établissement par les médias de «palmarès» non officiels des meilleurs services de police, en fonction des différents critères mesurés, mais aucune tentative plus scientifique d'utiliser les données disponibles afin d'identifier les meilleures pratiques organisationnelles à l'échelle nationale n'a encore vu le jour. En guise de conclusion, peut-être faut-il rappeler, au risque d'énoncer une tautologie, le caractère purement indicatif, voire approximatif, de ce mode d'évaluation. La sophistication de l'appareil méthodologique ne doit pas dissimuler ses aspects rudimentaires en comparaison de la complexité des systèmes étudiés. II offre la possibilité d'établir un diagnostic général de la santé de l'organisation policière à l'aide d'indicateurs de «première ligne», mais ne peut se substituer à des procédures plus détaillées d'évaluation axées sur des programmes ou des activités déterminés, par le biais d'indicateurs de "deuxième ligne» élaborés à l'aide de méthodes d'enquête sociologiques ou ethnographiques. Un outil hybride mariant ces deux démarches pourrait alors prétendre à une plus grande légitimité, tant auprès des services évalués et des usagers que des instances évaluatives elles-mêmes. 


\section{Références}

Bayley, D.H. (1994). Police for the future. N ew York : 0 xford University Press.

Bayley, D.H., \& Shearing, C. (2001). The new structure of policing: description, conceptualisation and research agenda. Washington, D.C. : N ational Institute of Justice.

Bennett, T., \& Lupton, R. (1992). A national activity survey of police work. The H oward Journal, 31 (3), 200-223.

Cheung, A.B.L. (1997). La compréhension des réformes du secteur public: tendances mondiales et questions diverses. Revue Internationale des Sciences Administratives, 63 (4), 513-537.

Crawford, A. (1998). Partenariat et responsabilité à l'ère managériale: retour sur I'expérience britannique. Les $C$ ahiers de la Sécurité Intérieure, 33, 51-87.

Ericson, R.V., \& H aggerty, K.D. (1997). Policing the risk society. 0 xford : Clarendon Press.

Grabosky, P. (1988). Efficiency and effectiveness in Australian policing. Canberra: Australian Institute of Criminology.

Kempa, M., Carrier, R., Wood, J., \& Shearing, C. (1999). Reflections on the evolving concept of «private policing». European Journal on Criminal Policy and Research, 7 (2), 197-224.

Loveday, B. (1993). M anagement and accountability in public services: a police case study. In K. I saac-H enry, C. Painter \& C. Barnes (eds), M anagement in the public sector : challenge and change (133-149). Londres: Chapman \& Hall.

Loveday, B. (1999). The impact of performance culture on criminal justice agencies in England and Wales. International Journal of the Sociology of Law, 27 (4), 351-377.

Loveday, B. (2000). M anaging crime: police use of crime data as an indicator of effectiveness. International Journal of the Sociology of Law, 28 (3), 215-237.

Moore, D. (1992). M easuring police productivity. In P. Moir \& H. Eijkman (eds), Policing Australia : old issues, new perspectives (40-66). M elbourne: M acmillan.

PA Consulting Group (2001). D iary of a police officer: Police research series paper 149. Londres: Home O ffice Policing and Reducing Crime Unit.

Pawson, R., \& Tilley, N. (1997). Realistic evaluation. Londres: Sage Publications.

Secretary of State (2002). T he police authorities (best value) performance indicators order 2002 : statutory instrument no. 694. Londres: Her M ajesty's Stationery 0 ffice.

Smith, P. (1992). N egative political feedback : an examination of the problem of modelling political responses in public sector effectiveness auditing. A ccounting, Auditing \& A ccountability Journal, 5 (1), 5-20.

Steering Committee for the Review of Commonwealth/ State Service Provision (1997). D ata Envel opment A nalysis: a techniquefor measuring the efficiency of government service delivery. C anberra: AGPS.

Steering Committee for the Review of Commonwealth/ State Service Provision (2002). Report on government services 2002. Canberra: Ausinfo. 
The Audit O ffice of N ew South Wales (1998). Performance audit report: N SW Police Service, police response to calls for assistance. Sydney: AO N SW.

Tilley, N. (1995). Thinking about crime prevention performance indicators: Crime detection \& prevention series paper 57. Londres: Home 0 ffice Police R esearch Group.

Waddington, P.A.J. (1999). Policing citizens. Londres: UCL Press.

Wilson, J.Q . (1993). The problem of defining agency success. In Performance measures for the criminal justice system. Washington, DC : B ureau of Justice Statistics. 